was removed shortly after surgery. Intravenous hydration was carried out for the first 6 hours postoperatively, followed by parenteral nutrition. Artificial enteral nutrition was started on the morning of postoperative day 1 , although this was interrupted in eight patients because of bowel distension or other complications.

The authors observed an accelerated recovery of bowel function in the group as a whole, which they attribute partly to reduced preoperative fasting, avoidance of mechanical bowel preparation, combined anesthesia and postoperative analgesia. Peristaltic movement returned more quickly in patients who had received artificial enteral nutrition.

The evaluation of the protocol is ongoing. Endpoints to be examined include the incidence of complications, length of hospital stay and patient satisfaction.

Original article Maffezzini M et al. (2004) Peri-operative management of ablative and reconstructive surgery for invasive bladder cancer in the elderly. Surg Oncol 13: 197-200

\section{Overactive bladder: improved quality of life using solifenacin}

According to a new study published in BJU International, solifenacin succinate-an oral antimuscarinic agent-significantly improves quality of life in patients with overactive bladder syndrome.

The study was based on the results of two randomized, 12-week trials comparing once daily 5 or $10 \mathrm{mg}$ solifenacin with placebo. The doubleblind period of each trial lasted 12 weeks and a total of nearly 2,000 patients were included. Results from 40-week, open-label extensions to both trials were also analyzed.

Patients' quality of life was assessed at baseline and at intervals throughout the study period using 10 domains of the King's Health Questionnaire. In separate analyses of both trials, patients receiving solifenacin showed significantly greater improvements, compared with placebo, in five of these domains. The combined results of the two trials, however, showed that patients on active treatment had significantly better scores than those on placebo for all domains except 'personal relationships'. Scores continued to improve throughout the open-label extension period. For patients who were randomized to placebo and then changed to solifenacin during the open-label extension, improvements were similar to those who had received the drug throughout.

Kelleher et al. note that the safety and efficacy of solifenacin for the treatment of overactive bladder syndrome has been demonstrated previously. Their analysis suggests that this therapy also provides long-term improvements in quality of life for these patients.

Original article Kelleher CJ et al. (2005) Improved quality of life in patients with overactive bladder symptoms treated with solifenacin. BJU Int 95: 81-85

\section{Acute urinary retention and quality of life}

A pilot study from the UK has shown that episodes of acute urinary retention (AUR) have a measurable and prolonged impact on healthrelated quality of life (HRQoL), particularly in terms of pain, hospital attendance and the need for extra help at home.

The study included 43 men aged over 50 years who presented to the accident and emergency department with AUR over a 2 year period. The impact of this event was assessed using a questionnaire derived from several established HRQoL instruments, including the Hospital Anxiety and Depression Score and the Medical Outcome Study Short-Form 12. For comparison, the investigators also recruited 35 men admitted for elective surgery for benign prostatic hyperplasia (BPH) and 17 men presenting with renal colic. Patients were invited to complete the questionnaire within 3 days of admission and again at 1, 2, 3 and 6 months.

Patients with AUR reported high pain scores (mean score 7.7 on a scale of $1-10$, compared with 5.6 for patients with BPH and 8.3 for those with renal colic), but pain quickly resolved after catheterization. Although AUR tended not to interfere greatly with employment, the need for assistance at home was greatest in this group, and this was associated with a considerable economic burden. Patients with AUR also reported more hospital admissions and specialized investigations than those in the other two groups during the study period.

Authors Thomas et al. conclude that there is a need to improve the 'patient journey' 